\title{
Correspondence
}

\section{Deliberate hypotension for intracranial aneurysm surgery: changing practices}

To the Editor:

I enjoyed Dr. Wong's review of perioperative stroke. ${ }^{1}$ However, the discussion of deliberate hypotension may leave readers with a misimpression. Dr. Wong states: "The risks of brain damage are minimal with deliberate hypotension provided that hypotensive anaesthesia is carefully administered." This statement follows mention of an investigation of CBF during nitroprusside-induced hypotension in aneurysm surgery. ${ }^{2}$ The measurements were made contralateral to the aneurysm in patients who were at least ten days post-subarachnoid haemorrhage, who were in good neurological condition, and who had no angiographic vasospasm. I believe this study was cited as confirmation that the normal brain is very tolerant of nitroprusside-induced hypotension. However, the juxtaposition of this information with the quoted statement may be misinterpreted as an endosement of deliberate hypotension during aneurysm clipping. By contrast, I believe that the evolving opinion of the neurosurgeryanaesthesia community is that it is preferable to minimize blood pressure reduction and to reserve hypotension for the control of intraoperative rupture or perhaps for brief periods preceding clip appiication.

This changing attitude is related in part to the recent practice of "early" clipping after subarachnoid haemorrhage and to the simultaneous development of an awareness of the brain's vulnerability in the post-injury period to damage by events that would, in isolation, be subcritical. This vulnerability to "secondary insult" by minor episodes of hypotension, hypoxia or ischaemia has been demonstrated in the laboratory for both previous ischaem$\mathrm{ic}^{3}$ and mechanical insult. ${ }^{4}$ Furthermore, subclinical vasospasm can occur. Patients of good neurological grade may have focal areas of substantial CBF reduction, ${ }^{5}$ and it is a logical concern that lowering perfusion pressure in these patients may cause reduction of CBF to critical levels. In addition, neurosurgeons increasingly use temporary occlusion to accomplish local decompression of technically difficult aneurysms ${ }^{6,7}$ and blood pressure may be critical in maintaining collateral flow.

The impact on neurological outcome of blood pressure management during aneurysm surgery has not been studied systematically. However, a recent review of intraoperative rupture concluded that patients in whom hypotension was used in the control of bleeding had poorer neurological outcome than those in whom normotension was maintained. ${ }^{8}$ This study may be inconclusive in that the use of hypotension may have correlated with more difficult technical circumstances. Nonetheless, it reflects an attitude of restraint in the use of hypotension in the care of these patients.

John C. Drummond MD FRCPC

University of California, San Diego and

VA Medical Center, San Diego

\section{REFERENCES}

1 Wong DH. Perioperative stroke. Part 1: General Surgery, carotid artery disease, and carotid endarterectomy. Can J Anaesth 1991; 38: 347-73.

2 Pinaud $M$, Souron $R$, Lelausque J-N, Gazeau M-F, Lajat $Y$, Dixneuf $B$. Cerebral blood flow and cerebral oxygen consumption during nitroprusside-induced hypotension to less than $50 \mathrm{mmHg}$. Anesthesiology 1989; 70: 255-60.

3 Tomida S, Nowak TS, Vass K, Lohr JM, Klatzo

I. Experimental model for repetitive ischemic attacks in the gerbil: the cumulative effect of repeated ischemic insults. J Cereb Blood Flow Metab 1987; 7 : 773-82.

4 Jenkins $L W$, Moszynski $K$, Lyeth $B G$ et al. Increased vulnerability of the mildly traumatized rat brain to cerebral ischemia: the use of controlled secondary ischemia as a research tool to identify common or different mechanisms contributing to mechanical and ischemic brain injury. Brain Res 1989; 477: 211-24.

5 Ishii $R$. Regional cerebral blood flow in patients with ruptured intracranial aneurysms. J Neurosurg 1979; 50: 587-94.

6 Young WL, Solomon RA, Pedley TA et al. Direct cortical EEG monitoring during temporary vascular occlusion for cerebral aneurysm surgery. Anesthesiology 1989; 71: 794-9.

7 Batjer HH, Frankfurt AL, Purdy PD, Smith SS, Samson $D S$. Use of etomidate, temporary arterial occlusion, and intraoperative angiography in surgical treatment of large and giant cerebral aneurysms. J Neurosurg 1988; 68: $234-40$.

8 Giannota $S L$, Oppenheimer JH, Levy ML, Zelman $V$. Management of intraoperative npture of aneurysm without hypotension. Neurosurgery 1991; 28: 531-36. 